Article

\title{
Synthesis of Antibacterial Silver-Poly( $\varepsilon$-caprolactone)-Methacrylic Acid Graft Copolymer Nanofibers and Their Evaluation as Potential Wound Dressing
}

\author{
Mohammed A. Al-Omair \\ Department of Chemistry, College of Science, King Faisal University, Al-Hassa 31982, Saudi Arabia; \\ E-Mail: alomair@kfu.edu.sa; Tel.: +966-50-390-0757
}

Academic Editor: Jung Kwon (John) Oh

Received: 21 May 2015 / Accepted: 29 July 2015 / Published: 12 August 2015

\begin{abstract}
Electrospun polycaprolacyone/polymethacrylic acid graft copolymer nanofibers (PCL/MAA) containing silver nanoparticles (AgNPs) were synthesized for effective wound disinfection. Surface morphology, AgNPs content, water uptake of electrospun PCL/MAA graft copolymer nanofibers without and with AgNPs, and levels of AgNPs leaching from the nanofibers in water as well as antimicrobial efficacy were studied. Scanning electron microscope images revealed that AgNPs dispersed well in PCL/MAA copolymer nanofibers with mean fiber diameters in the range of 200-579 $\mathrm{nm}$ and the fiber uniformity and diameter were not affected by the AgNPs. TEM images showed that AgNPs are present in/on the electrospun PCL/MAA graft copolymer nanofibers. The diameter of the electrospun nanofibers containing AgNPs was in the range of 200-579 nm, however, the diameter of AgNPs was within the range of 20-50 nm and AgNPs were observed to be spherical in shape. The PCL/MAA copolymer nanofibers showed a good hydrophilic property and the nanofibers containing AgNPs had excellent antimicrobial activity against the Gram-negative bacteria Escherichia coli and Pseudomonas aeruginosa, and against the Gram-positive bacteria Bacillus thuringiensis and Staphylococcus aureus, with a clear inhibition zone with a diameter between 22 and $53 \mathrm{~mm}$. Moreover, electrospun PCL/MAA copolymer nanofibers sustained the release of AgNPs into water over $72 \mathrm{~h}$.
\end{abstract}

Keywords: polycaprolacyone nanofibers (PCLNFs); PCLNFs-Silver nanocomposites; graft copolymer; electrospun nanofibers; functional nanofibers; wound dressing 


\section{Introduction}

Electrospinning is a unique nanofiber formation procedure in which nanofibers are molded by the application of electrical forces with a high voltage to yield nanofibers of different sizes, ranging from nano to a number of micrometers [1]. There are many factors that influence the synthesis of nanofibers by electrospinning. These include the practical voltage, the distance between the tip and the collector, the pumping rate of the polymeric solution, the polymer concentration, and the type of solvent used during the synthesis [2-4]. These nanofibers have many properties such as high porosity, large surface area, and the ability to facilitate gaseous exchange. These properties make them promising materials for different uses such as tissue engineering scaffolding, nanocatalysis, filtration, in the biomedical and pharmaceutical fields, and many other applications [5].

Furthermore, biodegradable polymers have attained significant consideration as biomaterials, which have medical applications in therapeutic and biomedical engineering uses [6,7]. Poly( $\varepsilon$-caprolactone) (PCL) is a domestic material of biodegradable aliphatic polyesters that has gained substantial use as a biomaterial. As a marketable material, the main attractive qualities of PCL are (i) the Food and Drug Administration (FDA) approval for use in human applications; (ii) biodegradability; (iii) compatibility with an extensive series of several polymers; (iv) good processability which allows the production of a variety of structures and forms; (v) ease of melt processing due to high thermal stability; and (vi) a relatively low cost $[8,9]$.

Wound dressings have several functions: they are used to protect the wound from external microbes, allow the release of excess exudate from the wound area, recover the look of the wound area, and occasionally speed up the therapeutic process. For these purposes, a wound bandage should deliver a substantial physical block to a wound, but should also be permeable to humidity and oxygen. Synthesized nanofiber mat by created by electrospinning is an excellent wound covering and dressing due to the presence of an extremely permeable network structure and good uniform holes. These characteristics are mostly significant for removing liquid from the wound. The minor pores and very large definite surface area not only prevent external microbial attacks, but also contribute in the control of fluid drainage. Moreover, the electrospinning procedure is comprised of a simple technique to enhance the incorporation of active ingredients into the nanofibers for any promising medicinal application and antiseptic determination [10].

Conventional dressings are not effective enough to achieve hemostasis, adherence, and maintenance of a moist environment around the wound [11]. Due to progress in the field of nanotechnology, it is now possible to design nanofiber-based wound dressings where an electrospun nanofibrous layer is applied to a basic support fabric material. These nanofibers have the following advantages [12]: (i) hemostasis occurs due to their nanofibrous structure; (ii) high filtration and liquid absorption efficiency of the nanofibers leads to high absorption efficiency of wound fluids; (iii) semi-permeability of nanofiber-based wound dressings facilitates cell respiration due to its porosity; (iv) conformability; (v) functional ability by loading antibiotics and analgesics through co-spinning; and (vi) scar-free result due to biodegradability. Furthermore, nanofiber-based wound dressings activate in moist environments, so there is no need to change the dressing every day, thus lessening pain and scars, which is extremely adequate for burn wounds. 
Histological tests show that the degree of epithelialization is improved and the dermis becomes well-controlled when the wounds are enclosed with the nanofiber mat. It was found that epidermal growth factor was immobilized into silk nanofiber mats to help the wound-healing process. The wound healing closer to these mats was increased by $90 \%$ [13]. An active ingredient with medical applications, curcumin, was incorporated into polycaprolactone [14] and cellulose acetate nanofibers [15] for wound dressings. These nanofiber dressings were more effective than the traditional dressings in healing dermal fibroblasts of human skin.

To disinfect the bacteria attack, numerous active ingredients such as silver [16,17] and iodine complex [18] have been studied. The immobilization of silver to polyvinyl alcohol nanofiber mats showed good antibacterial activity and enhanced a moist environment [16]. Iodine has been incorporated into polyvinylpyrrolidone solution to prepare iodine containing polyvinylpyrrolidone nanofiber mats and the slow release of iodine from the electrospun nanofibers has been studied [19]. Moreover, in the presence of antibacterial additives, biocidal electrospun nanofibers can also be developed directly via biopolymers. For example, electrospun polyurethane nanofibers with quaternary ammonium groups were prepared and showed reliable antimicrobial actions against Staphylococcus aureus and Escherichia coli [20].

The novelty of this article resides in the development of wound dressings based on controlled-release hydrophilic polycaprolactone nanofiber mats containing antibacterial nanoparticles which will be synthesized from poly-( $\varepsilon$-caprolactone)-polymethacrylic acid graft copolymer nanofibers loaded with AgNPs. The characterization of electrospun nanofiber mats using scanning electron microscopy, energy dispersive X-ray (EDX), transmission electron microscopy (TEM), the contents of AgNPs, the slow release of AgNPs, and water uptake as well as antibacterial activity will be studied.

\section{Experimental Methods}

\subsection{Materials}

Poly- $\varepsilon$-caprolactone (PCL) (65 kDa), silver nitrate as a precursor, Dichloromethane (DCM) as a co-solvent, and benzoyl peroxide as an initiator were purchased from Sigma Aldrich (St. Louis, MO, USA). Methacrylic acid (MAA) monomer was purchased from Sigma-Aldrich Co. (St. Louis, MO, USA). All reagents used in this study were of analytical grade.

\subsection{Grafting Copolymerization of MAA on to Polycaprolactone}

Poly( $\varepsilon$-caprolactone) solution (PCL) was prepared by dissolving $5 \mathrm{~g}$ PCL in $5 \mathrm{~mL}$ dichloromethane and stirring until the PCL was completely dissolved and then $20 \mathrm{~mL}$ of ethanol was added under constant stirring for $2 \mathrm{~h}$ in a $250 \mathrm{~mL}$ round bottom flask. Final volume of the solution was made up to $100 \mathrm{~mL}$ by the addition of ethanol. Methacrylic acid (MAA) 50\% (w/v) of the final solution was added and then benzyl peroxide was used as an initiator, at a concentration of $0.5 \mathrm{wt} \%$ of MAA. Solution was transferred into polyethylene tube with $10 \mathrm{~mm} \times 200 \mathrm{~mm}$ internal diameter and length, respectively. Nitrogen gas was used to remove the air or oxygen from the solution for 15-20 min. The covered tube was located in the water bath at $50{ }^{\circ} \mathrm{C}$ for $2 \mathrm{~h}$. After the reaction time, the tube was removed and cooled to room temperature and the prepared grafted copolymer was removed from the tube. It was transferred to a 
reaction thimble and washed with 50\% v/v ethanol/water mixture using a Soxhlet apparatus for 5 days, for complete removal of the un-reacted monomers. The prepared graft copolymer was dried, first at room temperature and then in an oven at $40{ }^{\circ} \mathrm{C}-45^{\circ} \mathrm{C}$ for 1 week. Graft yield was $58 \%$.

\subsection{Synthesis of PCL Nanofibers and PCL/MAA Nanofibers Containing AgNPs}

A polymer solution of PCL/MAA graft copolymer was prepared by dissolving it in dichloromethane $(14 \% \mathrm{w} / \mathrm{v})$. The prepared solution was placed in a water bath under stirring conditions for $24 \mathrm{~h}$ at $25{ }^{\circ} \mathrm{C}$ to $65{ }^{\circ} \mathrm{C}$, with a heating rate of $5{ }^{\circ} \mathrm{C} / \mathrm{min}$. After this, $5 \%(w / v) \mathrm{AgNO}_{3}$ was thoroughly mixed into the polymer solution. Electrospinning of the prepared solution was carried out using NEU-010 Nanofiber Electrospinning Unit (Kes Kato Tech Co., Tokyo, Japan). High voltage direct current-power supply was applied to the standard $20 \mathrm{~mL}$ syringe containing the polymer solution, the open end of which was attached to a blunt gauge-20 stainless steel needle, used as the nozzle. A fixed electrical potential of $20 \mathrm{kV}$ was applied across a fixed distance of $10 \mathrm{~cm}$ between the tip of the nozzle and the outer surface of the target drum, which was used as a nanofiber collector. Rotational speed of the rotating drum was kept at $40 \pm 5 \mathrm{r} / \mathrm{min}$ and the feed rate of the solution was controlled by a syringe pump at $\sim 1 \mathrm{~mL} / \mathrm{h}$. Electrospinning process was carried out under laboratory conditions (i.e., temperature $=25{ }^{\circ} \mathrm{C} \pm 1{ }^{\circ} \mathrm{C}$; relative humidity $=71 \% \pm 3 \%$ ). Electrspun PCL nanofibers containing $\mathrm{AgNO}_{3}$ were collected on a plate. Collected nanofibers were immersed in sodium borohydride solution of $0.5 \mathrm{wt} \%(w / v)$ for $1 \mathrm{~h}$ to reduce any silver ions residual in the nanofibers of AgNPs.

\subsection{Characterization of PCL/MAA Nanofibers Containing AgNPs}

The morphology of electrospun PCL/MAA copolymer nanofibers containing AgNPs was studied using a field emission scanning electron microscope coupled with energy dispersive X-ray analysis (EDX) (JOEL SM7600F-USA, Pleasanton, Canada). Additional elemental analysis and quantification of silver in the fibers was done with EDX. The actual AgNPs content was determined by atomic absorption spectroscopy (Varian SpectrAA-220 Zeeman, Mulgrave, Australia) with a graphite furnace accessory and equipped with deuterium arc background corrector according to the manufacturer's instructions (APHA 2005). Appropriate amounts of electrospun nanofibers containing AgNPs were digested in $\mathrm{HNO}_{3}(1 \mathrm{M})$ for $24 \mathrm{~h}$ at room temperature. The average diameter of the AgNPs on the electrospun PCL/MAA copolymer nanofibers was determined using transmission electron microscopy (TEM) (Carl Zeiss EM 10, Oberkochen, Germany).

\subsection{Atomic Absorption Analysis of AgNPs Leaching from PCL/MAA Electrospun Nanofibers}

The release of AgNPs from PCL/MAA nanofibers was assessed according to the manufacturer (APHA 2005) by determining its concentration in the non-solvent dipping bath by atomic absorption (Spectra AA 220, Varian Inc., Zeeman, Australia, with graphite furnace attachment and prepared with deuterium arc background corrector) with a hollow cathode Ag lamp at $328.1 \mathrm{~nm}$. Nanofiber samples containing AgNPs were transferred to shaking bottles with $10 \mathrm{~mL}$ deionized water. The bottles then were sealed with para-film, covered and secured, rotated end-over-end for different times (1-24 h) at $30 \mathrm{rpm}$ and then filtrated through $0.45 \mu \mathrm{m}$ membrane filters. Released AgNPs were analyzed in the filtrate. 


\subsection{Water Uptake Measurement (WU)}

To measure the degree of swelling of the electrospun PCL/MAA copolymer nanofibers with and without AgNPs, electrospun nanofibers were placed in a small glass dish and weighed (dry weight). The samples were suspended in $10 \mathrm{~mL}$ of deionized water. The dish was covered with para-film and stored at room temperature. After a specific duration, the liquid was drained and the nanofiber mats were removed from the dish. The nanofiber mats were gently shaken to remove any excess water, and then weighed (wet weight). Water uptake was calculated using the following equation:

$$
W U=\frac{W W-D W}{D W} \times 100
$$

where $W W$ is the wet weight and $D W$ is dry weight.

\subsection{Antimicrobial Activity of Electrospun PCL/MAA Copolymer Nanofibers Containing AgNPs}

The antimicrobial investigation of electrospun PCL/MAA graft copolymer nanofibers with and without AgNPs was carried out using diffusion technique [18]. The experiment was carried out against gram-negative bacteria such as Escherichia coli and Pseudomonas aeruginosa, and against gram-positive bacteria such as Bacillus thuringiensis and Staphylococcus aureus. The Luria-Bertani Agar (LBA) medium (10 g bacto-tryptone, $5 \mathrm{~g}$ yeast extract, $20 \mathrm{~g}$ agar, and $10 \mathrm{~g} \mathrm{NaCl}$ in 1 liter de-ionized water) was prepared for bacterial development. The tested electrospun PCL/MAA graft copolymer nanofibers with and without AgNPs were located individually in the agar. The LBA plates were incubated for $24 \mathrm{~h}$ at $37{ }^{\circ} \mathrm{C}$ and the resulting clear inhibition zones were determined. The antimicrobial activity against the gram-negative and gram-positive bacteria was identified by the clear inhibition zone diameter.

\section{Results and Discussion}

\subsection{Characterization of Electrospun PCL/MAA Graft Copolymer Nanofibers}

Figure 1a shows the scanning electron micrograph at a magnification $45,000 \times$ of electrospun PCL/MAA graft copolymer nanofibers without AgNPs, which reveals uniformly distributed nanofibers with mean fiber diameters in the range of 200-579 $\mathrm{nm}$. Figure 1b shows the SEM micrograph of the electrospun PCL/MAA graft copolymer nanofibers with AgNPs and the image reveals that the presence of silver nanofibers did not affect the fiber uniformity and diameter. As shown in Figure 1b, pure PCL/MAA nanofiber produce smooth and bead-free nanofibers with a diameter ranging from 200 to $578 \mathrm{~nm}$.

Figure 2 shows the energy dispersive spectrum (EDX) collected on the PCL/MAA containing AgNPs. The sample clearly identifies Ag as the elemental component in the nanofibers. The other peaks belonging to carbon are generated from the PCL. Elementary analysis of PCL/MAA containing AgNPs nanofibers was carried out by using SEM-EDX. The results show that carbon and Ag were the principal elements of PCL/MAA/AgNPs nanofibers. EDX analysis proves direct evidence that AgNPs is embedded in the PCL/MAA nanofibers. It also indicated that AgNPs are well loaded without any chemical and structural modifications into the PCL/MAA graft copolymer. 


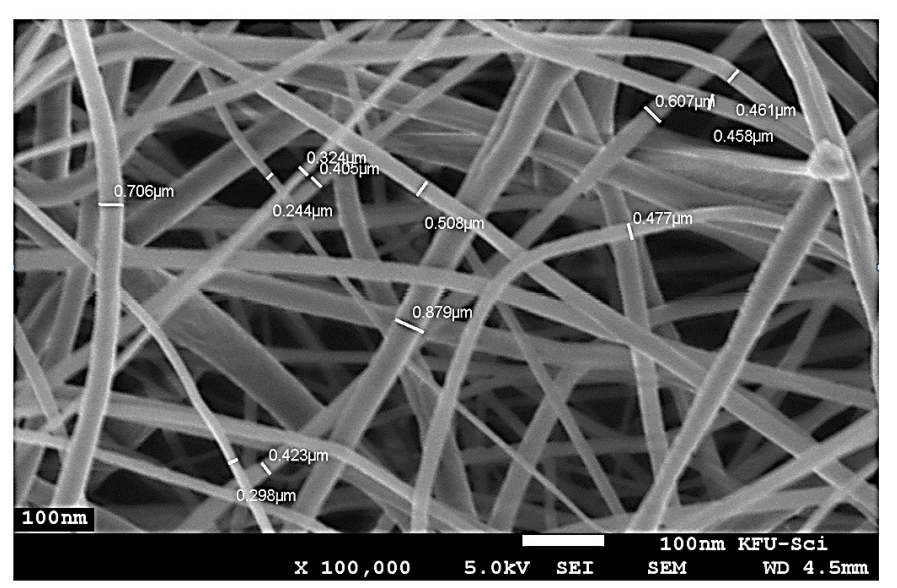

a

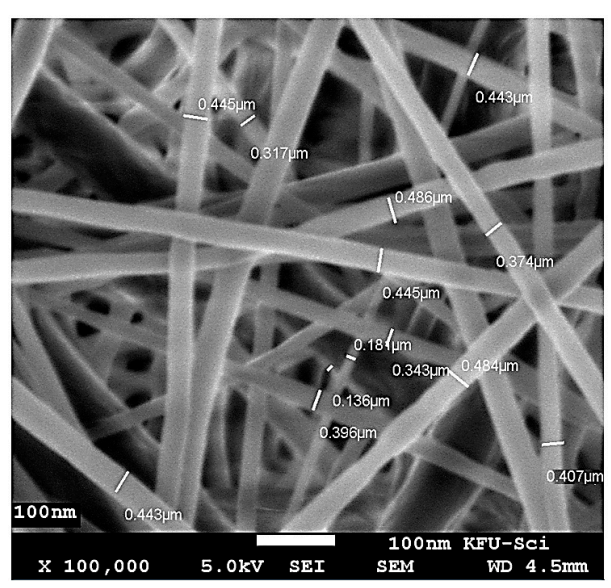

b

Figure 1. Scanning electron microscopy of PCL/MAA $(14 \% w / v)$ graft copolymer nanofibers (a); and PCL/MAA graft copolymer nanofibers containing silver nitrate 5\% (b).

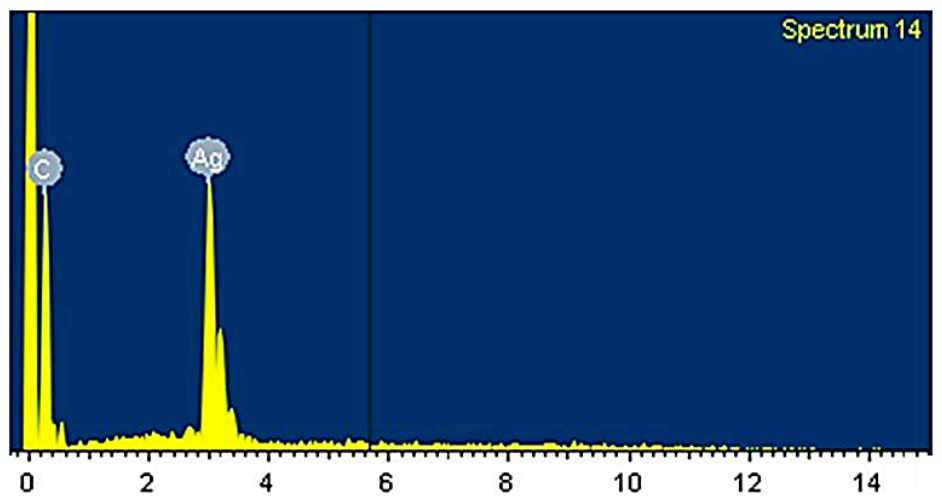

Figure 2. Energy dispersive X-ray collected on PCL/MAA nanofibers containing AgNPs.

\subsection{TEM Image of Electrospun PCL/MAA Copolymer Containing AgNPs}

Figure 3 shows a high magnification power $(500,000 \times)$ transmission electron microscopy image of electrospun PCL/MAA copolymer nanofibers containing AgNPs. It can be noticed that AgNPs are present in/on the electrospun PCL/MAA graft copolymer nanofibers. Generally, the diameter of the electrospun nanofibers was in the range of 200 to $578 \mathrm{~nm}$, however, the diameter of AgNPs was within the range of 20 to $50 \mathrm{~nm}$ and AgNPs can be seen as a spherical shape. Likewise, it can be observed that when the TEM image is compared with SEM images, the nanoparticle concentration is higher in the TEM image than in SEM. Knowing the fact that the electron beam in TEM examination passes within the nanofibers, it can be observed that the AgNPs were integrated inside the nanofibers. Nevertheless, in the case of SEM, only the surface morphology is examined. However, TEM images show the difference between the electrospun nanofibers with and without AgNPs; in the TEM image in Figure 3b, the nanoparticles are clearly distinguished (the dark spots). 


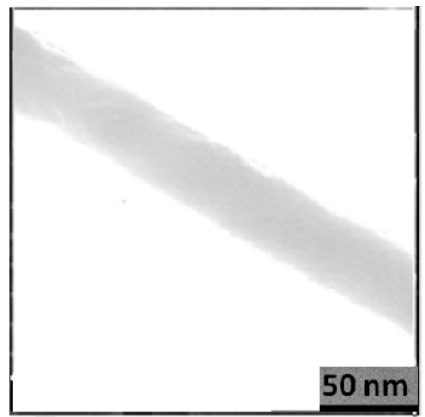

(a)

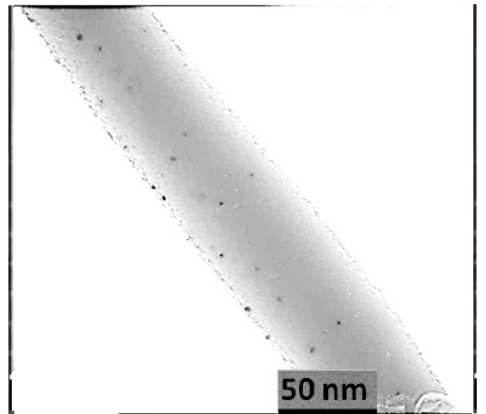

(b)

Figure 3. TEM image of electrospun PCL/MAA graft copolymer nanofiber without AgNPs (a) and containing AgNPs (b).

\subsection{Water Uptake of Electrospun PCL/MAA Copolymer Nanofibers}

The absorbance capacity is represented by the swelling degree of nanofibers in water uptake. Figure 4 shows the water uptake of electrospun PCL/MAA graft copolymer nanofibers, PCL/MAA graft copolymer nanofibers containing AgNPs, and electrospun PCL nanofibers. The data reveals that the water uptake of electrospun nanofibers has the following decreasing order: PCL/MAA nanofibers > PCL/MAA-AgNPs nanofibers > PCL nanofibers. This is attributed to the presence of hydrophilic carboxyl groups located in the polymer backbone. The hydrophilicity slightly decreased due to the incorporation of AgNPs, while in the case of PCL nanofibers, the hydrophobicity increased. On the other hand, in the case of PCL/MAA graft copolymer nanofibers, the amount of water uptake increased within $1 \mathrm{~min}$ and more than doubled after three days.

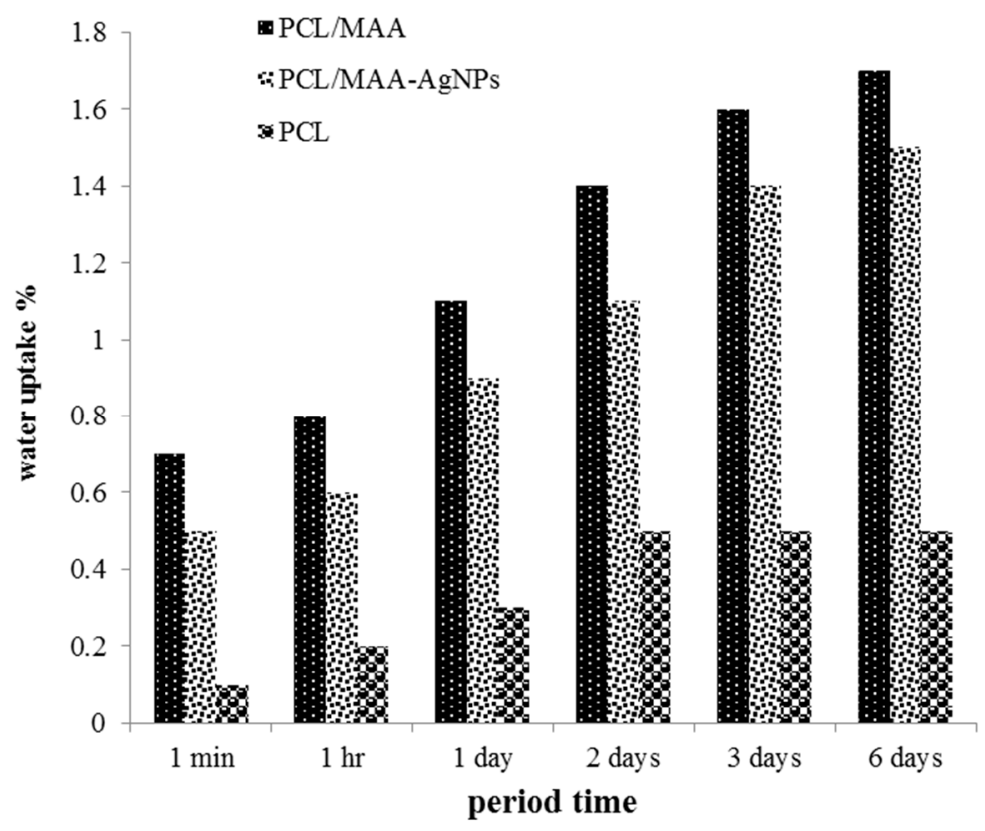

Figure 4. Water uptake (\%) of electronspun PLC/MAA graft copolymer, PCL/MAA-AgNPs and PCL nanofibers. 


\subsection{Leaching of AgNPs from PCL/MAA Graft Copolymer Nanofibers}

The leaching of AgNPs from PCL/MAA graft copolymer nanofibers was examined after soaking these nanofibers in water for different durations of time. The leaching experiment was performed by the measurement of AA spectra of AgNPs in the water used for soaking the samples. Table 1 shows the leached AgNPs from AgNPs-loaded PCL/MAA electrospun nanofiber mats during different periods of time: $1,4,10,24,48$, and $72 \mathrm{~h}$. It is observed that in the initial $1 \mathrm{~h}$, the AgNPs release rate increases because of lightly adhered AgNPs and the initial burst release. Then, it is followed by a slow release driven by the diffusion of AgNPs through PCL/MAA electrospun nanofibers. The release of AgNPs from the mat occurs within a range of $10-20 \mathrm{mg} / 100 \mathrm{~mL}$. The study shows a constant release of AgNPs over the whole period of $72 \mathrm{~h}$. UV spectroscopy was used to confirm the release of silver in the form of nanoparticles. Figure 5 shows a single strong peak at $418 \mathrm{~nm}$, which corresponds to the typical surface plasmon resonance (SPR) of AgNPs [21]. There are no peaks located around 335 and $560 \mathrm{~nm}$, indicating the complete absence of silver ions and any particle aggregates [22].

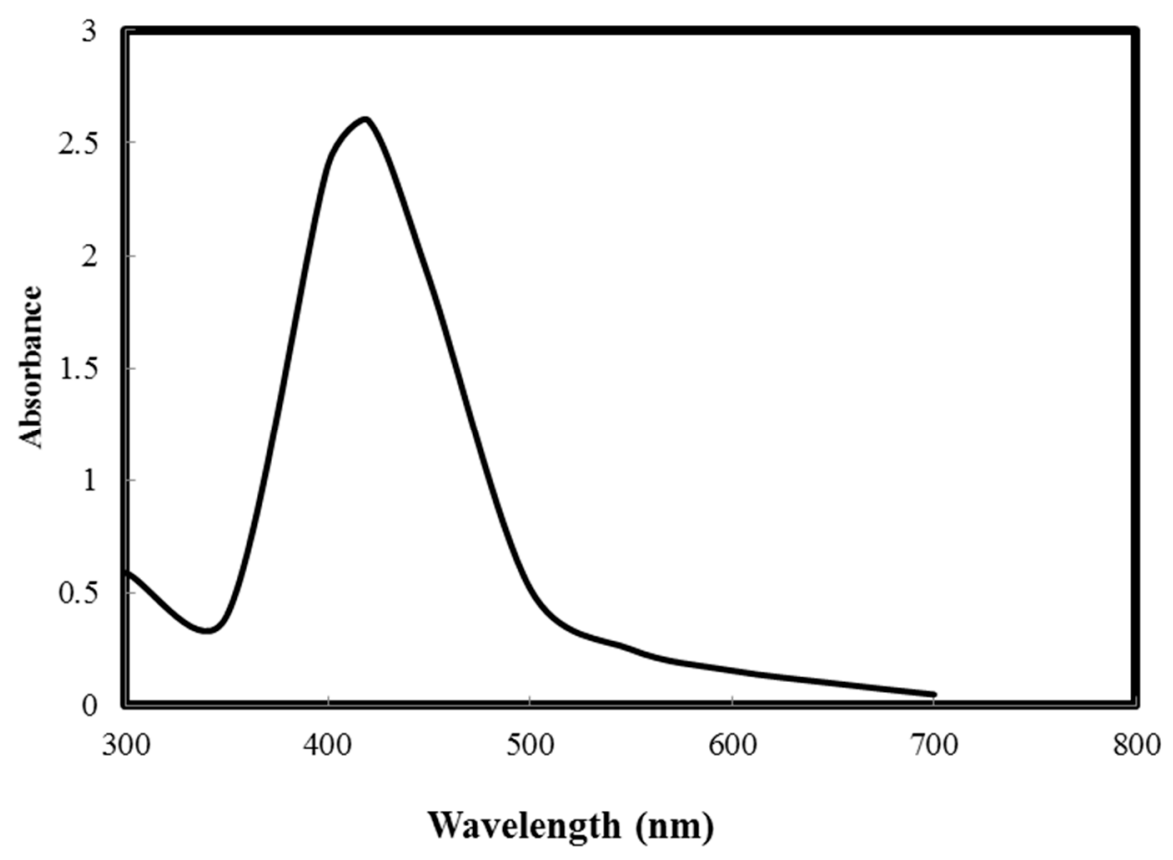

Figure 5. UV-visible absorption spectra of AgNPs.

Table 1. Leaching of AgNPs from PCL/MAA graft copolymer nanofibers containing AgNPs.

\begin{tabular}{cc}
\hline Release Time (h) & Leaching Silver Concentration $\mathbf{~ m g / 1 0 0 ~ g}$ \\
\hline 0 & 70 \\
1 & 50 \\
4 & 30 \\
10 & 25 \\
24 & 20 \\
48 & 15 \\
72 & 10 \\
\hline
\end{tabular}




\subsection{Antibacterial Activity of Electrospun PCL/MAA Containing AgNPs}

Nanofibers have a wide range of medical applications, especially in the treatment of wounds because of their distinctive characteristics. The porous composition and the connection between the pores play an important role in releasing the fluids from wounds. The high specific surface area not only inhibits the external microbe attacks, but also assists the control of fluid drainage. The antimicrobial activity of PCL/MAA-AgNPs was investigated as described in Figure 6 and Table 2. It was found that electrospun PCL/MAA copolymer nanofibers containing AgNPs had higher antimicrobial activity against the gram-negative bacterial strains Escherichia coli and Pseudomonas aeruginosa and gram-positive bacterial strains Bacillus thuringiensis and Staphylococcus aureus than the electrospun PCL/MAA graft copolymer nanofibers without AgNPs. Antibacterial activity of electrospun PCL/MAA-AgNPs against gram-positive bacteria is higher than the gram-negative bacteria. The clear inhibition zone diameter of PCL/MAA-AgNPs against the bacteria has the following order: Staphylococcus aureus $>$ Bacillus thuringiensis $>$ Pseudomonas aeruginosa $>$ Escherichia coli. In general, these nanofibers showed very strong antibacterial activity against the tested human pathogenic bacteria. It could be concluded that the electrospun grafted copolymer nanofiber systems loaded with AgNPs can potentially be used as an effective antibacterial wound dressing.
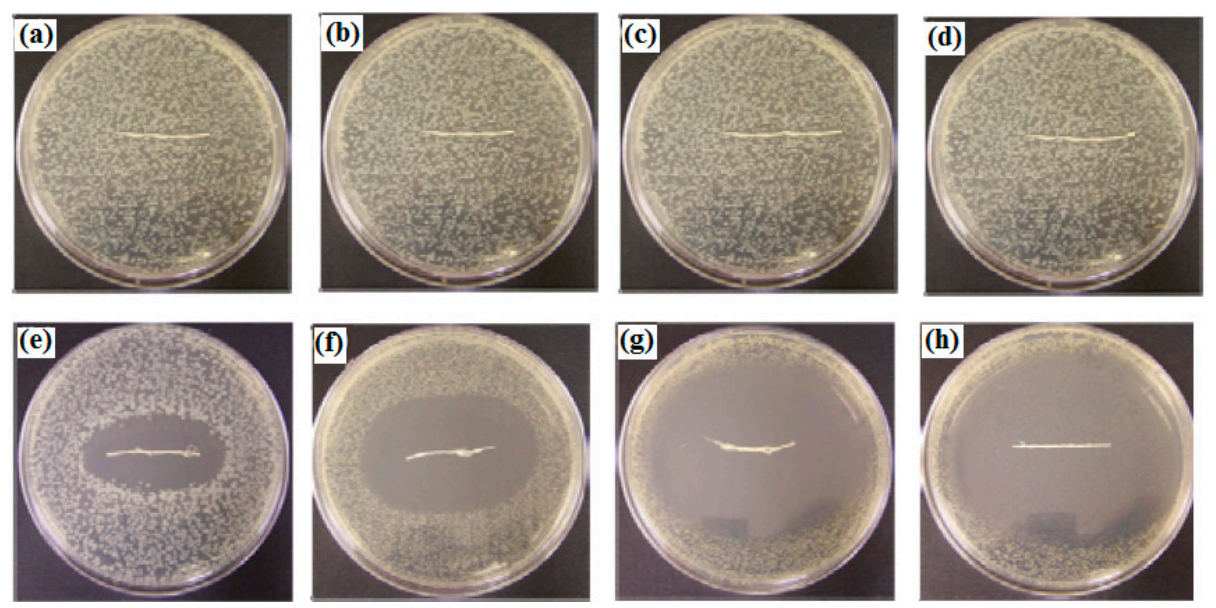

Figure 6. Clear inhibition zone diameter of electrospun PCL/MAA copolymer nanofibers without AgNPs against (a) Escherichia coli; (b) Pseudomonas aeruginosa; (c) Bacillus thuringiensis; (d) Staphylococcus aureus; and electrospun PCL/MAA copolymer nanofibers containing AgNPs against; (e) Escherichia coli; (f) Pseudomonas aeruginosa; (g) Bacillus thuringiensis; and (h) Staphylococcus aureus.

Table 2. Clear inhibition zone diameter $(\mathrm{mm})$ of electrospun PCL/MAA graft copolymer nanofibers without and with AgNPs against human pathogenic bacteria.

\begin{tabular}{cccccc}
\hline \multirow{2}{*}{ Sample } & \multicolumn{4}{c}{ Clear Inhibition Zone Diameter (mm) } \\
\cline { 2 - 3 } \cline { 5 - 6 } & \multicolumn{2}{c}{ Gram negative bacteria } & & \multicolumn{2}{c}{ Gram positive bacteria } \\
\cline { 2 - 3 } \cline { 5 - 6 } & Escherichia coli & Pseudomonas aeruginosan & & Staph. aurous & Bacillus thuringiensis \\
\hline PCL/MAA & 0 & 0 & & 0 & 0 \\
PCL/MAA-AgNPs & 22 & 26 & & 53 & 50 \\
\hline
\end{tabular}




\section{Conclusions}

Electrospun PCL/MAA graft copolymer nanofiber mats containing AgNPs were successfully prepared using the electrospinning technique. Major conclusions from these studies are given below:

The SEM images of the electrospun nanofiber mats show well-rounded fibers in a good network shape. These fibers are smooth and have average diameters of 200-579 nm. The TEM image shows that AgNPs are present in/on the electrospun PCL/MAA graft copolymer nanofibers. The diameter of the electrospun nanofibers containing AgNPs was in the range of 200-579 nm, however, the diameter of AgNPs was within the range of 20-50 nm and AgNPs can be seen as a spherical shape. The water uptake of electrospun nanofibers has the following decreasing order: PCL/MAA nanofibers > PCL/MAA-AgNPs nanofibers > PCL nanofibers. The hydrophilicity slightly decreased due to the incorporation of AgNPs, while in the case of PCL nanofibers, the hydrophobicity increased. On the other hand, in the case of PCL/MAA graft copolymer nanofibers, the amount of water absorbance increased within 1 min and more than doubled after three days.

In the in vitro release study, the rate of the AgNPs release is characterized by an initial release in the first $4 \mathrm{~h}$ followed by a sustainable release during the period of 10-72 $\mathrm{h}$. Electrospun PCL/MAA graft copolymer nanofibers containing AgNPs had higher antimicrobial activity against the gram-negative bacterial strains Escherichia coli and Pseudomonas aeruginosa and against gram-positive bacterial strains Bacillus thuringiensis and Staphylococcus aureus than the electrospun PCL/MAA graft copolymer nanofibers without AgNPs.

\section{Acknowledgments}

I would like to thank King Faisal University for its continuous support, encouragement and faciltation of research making.

\section{Conflicts of Interest}

The author declares no conflict interest.

\section{References}

1. New, N.; Furuike, T.; Tamura, H. The mechanical and biological properties of chitosan scaffolds for tissue regeneration templates are significantly enhanced by chitosan from Gongronella butleri. Materials 2009, 2, 374-398.

2. Tran, D.L.; Pham, G.D.; Nguyen, X.P.; Vu, D.H.; Nguyen, N.T.; Tran, V.H.; Mai, T.T.T.; Nguyen, H.B.; Le, Q.D.; Nguyen, T.N.; et al. Some biomedical applications of chitosan-based hybrid nanomaterials. Adv. Nat. Sci. Nanosci. Nanotechnol. 2011, 2, 45004. [CrossRef]

3. El-hefian, E.A. Characterization of chitosan in acetic acid: Rheological and thermal studies. Turk. J. Chem. 2010, 34, 47-56.

4. Aranaz, I.; Mengíbar, M.; Harris, R. Functional characterization of chitin and chitosan. Curr. Chem. Biol. 2009, 3, 203-230.

5. Lee, D.W.; Lim, H.; Chong, H.N.; Shim, W.S. Advances in chitosan material and its hybrid derivatives: A review. Open Biomater. J. 2009, 1, 10-20. [CrossRef] 
6. Jayakumar, R.; Prabaharan, M.; Nair, S.V.; Tamura, H. Novel chitin and chitosan nanofibers in biomedical applications. Biotechnol. Adv. 2010, 28, 142-150. [CrossRef] [PubMed]

7. Bikiaris, D.N.; Papageorgiou, G.Z.; Achilias, D.S.; Pavlidou, E.; Stergiou, A. Miscibility and enzymatic degradation studies of poly( $\varepsilon$-caprolactone)/poly-(propylene succinate) blends. Eur. Polym. J. 2007, 43, 2491-2503. [CrossRef]

8. Charuchinda, A.; Molly, R.; Siripitayananon, J.; Molloy, N.; Sriyai, M. Factors influencing the small-scale melt spinning of poly( $\varepsilon$-caprolactone) monofilament fibers. Polym. Int. 2003, 52, 1175-1181. [CrossRef]

9. Cipitria, A.; Skelton, A.; Dargaville, T.R.; Dalton, P.D.; Hutmacher, D.W. Design, fabrication and characterization of PCL electrospun scaffolds-A review. J. Mater. Chem. 2011, 21, 9419-9453. [CrossRef]

10. Khil, M.S.; Cha, D.I.; Kim, H.Y.; Kim, I.S.; Bhattarai, N. Electrospun nanofibrous polyurethane membrane as wound dressing. J. Biomed. Mater. Res. B 2003, 67, 675-679. [CrossRef] [PubMed]

11. Alves, J.L.; Bellino, N.J.; Geraldes, M.J. Nano-bioabsorbent composite wound dressing for exudate management. WIREs Nanomed. Nanobiotechnol. 2010, 2, 510-525.

12. Zahedi, P.; Rezaeian, I.; Ranaei-Siadat, S.; Jafari, S.; Supaphol, P. A review on wound dressings with an emphasis on electrospun nanofibrous polymeric bandages. Polym. Adv. Technol. 2010, 21, 77-95. [CrossRef]

13. Kang, Y.O. Chitosan-coated poly(vinyl alcohol) nanofibers for wound dressings. J. Biomed. Mater. Res. B 2010, 92, 568-576. [CrossRef] [PubMed]

14. Schneider, A.; Wang, X.Y.; Kaplan, D.L.; Garlick, J.A.; Egles, C. Biofunctionalized electrospun silk mats as a topical bioactive dressing for accelerated wound healing. Acta Biomater. 2009, 5, 2570-2578. [CrossRef] [PubMed]

15. Merrell, J.G.; McLaughlin, S.W.; Tie, L.; Laurencin, C.T.; Chen, A.F.; Nair, L.S. Curcumin-loaded poly( $\varepsilon$-caprolactone) nanofibers: Diabetic wound dressing with anti-oxidant and anti-inflammatory properties. Clin. Exp. Pharmacol. Physiol. 2009, 36, 1149-1156. [CrossRef] [PubMed]

16. Suwantong, O.; Ruktanonchai, U.; Supaphol, P. In vitro biological evaluation of electrospun cellulose acetate fiber mats containing asiaticoside or curcumin. J. Biomed. Mater. Res. A 2010 , 94, 1216-1225. [PubMed]

17. Hong, K.H. Preparation and properties of electrospun poly(vinyl alcohol)/silver fiber web as wound dressings. Polym. Eng. Sci. 2007, 47, 43-49. [CrossRef]

18. Chen, J.; Chiang, Y. Bioactive electrospun silver nanoparticles-containing polyurethane nanofibers as wound dressings. J. Nanosci. Nanotechnol. 2010, 10, 7560-7564. [CrossRef] [PubMed]

19. Ignatova, M.; Manolova, N.; Rashkov, I. Electrospinning of poly(vinyl pyrrolidone)-iodine complex and poly(ethylene oxide)/poly(vinyl pyrrolidone)-iodine complex-A prospective route to antimicrobial wound dressing materials. Eur. Polym. J. 2007, 43, 1609-1623. [CrossRef]

20. Kenawy, E.-R.; Bowlin, G.L.; Mansfield, K.; Layman, J.; Simpson, D.G.; Sanders, E.H.; Wnek, G.E. Release of tetracycline hydrochloride from electrospun poly(ethylene-co-vinylacetate), poly(lactic acid), and a blend. J. Control. Release 2002, 81, 57-64. [CrossRef] 
21. Sosa, I.O.; Noguez, C.; Barrera, R.G. Optical properties of metal nanoparticles with arbitrary shapes. J. Phys. Chem. B 2003, 107, 6269-6275. [CrossRef]

22. Murthy, P.S.K.; Moha, Y.M.; Varaprasa, K.D.; Sreedhar, B.; Raju, K.M. First successful design of semi-IPN hydrogel-silver nanocomposites: A facile approach for antibacterial application. J. Colloid Interface Sci. 2008, 318, 217-224. [CrossRef] [PubMed]

(C) 2015 by the author; licensee MDPI, Basel, Switzerland. This article is an open access article distributed under the terms and conditions of the Creative Commons Attribution license (http://creativecommons.org/licenses/by/4.0/). 